\title{
Um resgate histórico do LABIOMEC na história do CEFD 50 anos: parte 2
}

\author{
A historical rescue of the LABIOMEC in the history of CEFD 50 years: parte 2
}

Un rescate histórico del LABIOMEC en la historia de CEFD 50 años: part 2

Carlos Bolli Mota ${ }^{\mathrm{I}}$, Juliana Corrêa Soares ${ }^{\mathrm{II}}$, Carla Emilia Rossato ${ }^{\mathrm{III}}$, Franciele Marques Pivetta ${ }^{\mathrm{IV}}$, Marta Cristina Rodrigues da Silva ${ }^{\mathrm{V}}$, Mateus Corrêa Silveira ${ }^{\mathrm{VI}}$, Gabriela dos Santos de Souza ${ }^{\mathrm{VII}}$, Estele Caroline Welter Meereis-Lemos ${ }^{\mathrm{VIII}}$, Igor Martins Barbosa ${ }^{\mathrm{IX}}$, Rose Löbell ${ }^{\mathrm{X}}$, Rafaela Oliveira Machado ${ }^{\mathrm{XI}}$, Michele Forgiarini Saccol ${ }^{\mathrm{XII}}$

\section{Resumo}

Este trabalho tem como objetivo relatar um pouco da história e da evolução do Laboratório de Biomecânica (LABIOMEC) ao longo dos 50 anos do Centro de Educação Física e Desportos (CEFD) da Universidade Federal da Santa Maria. Para tanto foram realizadas buscas em documentos físicos e arquivos digitais no período de 1970 até os dias atuais em relação ao LABIOMEC em artigos, trabalhos em eventos, monografias de graduação, especialização, dissertações e teses. $\mathrm{O}$ artigo foi subdividido em duas partes e nesta segunda é realizado o resgate histórico da evolução técnico-científica ocorrida nas linhas de pesquisa da cinemática e cinética, controle postural e eletromiografia, encerrando com as atividades de divulgação científica realizadas no National Biomechanics Day. A importância desse relato é permitir que parte da história do LABIOMEC e das pessoas que realizaram suas atividades no laboratório como docentes ou discentes fique registrada de forma permanente.

Palavras-chave: Biomecânica; Educação Física e Treinamento; Movimento

\begin{abstract}
The purpose of this work is to report a little of the history and evolution that the Laboratory of Biomechanics (LABIOMEC) experienced over the 50 years of the Center for Physical Education and Sports (CEFD) of the Federal University of Santa Maria. For that purpose, searches were carried out on physical documents and digital files from 1970 to the present day in relation to LABIOMEC in articles, works in events, graduation monographs, specialization, dissertations and theses. The article was subdivided into two parts and in this second one, the historical recovery of the technical-scientific evolution occurred in research areas of kinematics and kinetics, postural control and electromyography, ending with the activities of scientific dissemination during the National Biomechanics Day. The importance of this report is to allow part of the history of LABIOMEC and the people who carried out their activities in the laboratory as teachers or students to be permanently registered.
\end{abstract}

Keywords: Biomechanics; Physical Education and Training; Movement

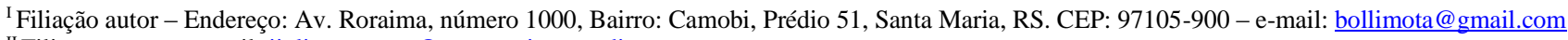

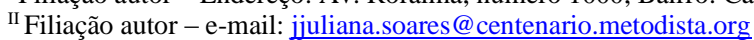

III Filiação autor - e-mail: carlinharossato@gmail.com

${ }^{\mathrm{IV}}$ Filiação autor - e-mail: fran87.mp@ gmail.com

v Filiação autor - e-mail: martacrys1605@gmail.com

${ }^{V I}$ Filiação autor - e-mail: mm.biomec@gmail.com

VII Filiação autor - e-mail: ggabrielassouza@gmail.com

VIII Filiação autor - e-mail: estelemeereis@gmail.com

IX Filiação autor - e-mail: igormartinsbarbosa2@gmail.com

x Filiação autor - e-mail: roselobell@gmail.com

XI Filiação autor - e-mail: rafa omachado@hotmail.com

XII Filiação autor - e-mail: $\underline{\text { mfsaccol@gmail.com }}$
} 


\section{Resumen}

El propósito de este trabajo es informar un poco de la historia y evolución que experimentó el Laboratorio de Biomecánica (LABIOMEC) durante los 50 años del Centro de Educación Física y Deportes (CEFD) de la Universidad Federal de Santa María. Para ello, se realizaron búsquedas en documentos físicos y archivos digitales desde 1970 hasta la actualidad en relación con LABIOMEC en artículos, trabajos en eventos, monografías de graduación, especialización, disertaciones y tesis. El artículo fue subdividido en dos partes y em esta segunda se realiza la recuperación histórica de la evolución técnico-científica ocurrida em las areas de investigación en cinemática y cinética, control postural y electromiografía, culminando com las actividades de divulgación científica realizadas en el Día Nacional de la Biomecánica. La importancia de esto El informe es para permitir que parte de la historia de LABIOMEC y las personas que llevaron a cabo sus actividades en el laboratorio como maestros o estudiantes se registren permanentemente.

Palabras clave: Biomecánica; Educación y Entrenamiento Físico; Movimiento

\section{Introdução}

A parte 1 desse artigo apresentou parte do resgaste histórico do Laboratório de Biomecânica (LABIOMEC) que ocorreu simultaneamente ao desenvolvimento da biomecânica no país, passando por sua criação como um núcleo dentro do Laboratório de Pesquisa e Ensino do Movimento Humano (LAPEM) e o desenvolvimento técnico-científico que foi necessário naquele período para o desenvolvimento das atividades de pesquisa.

A partir da década de 90, a aquisição de equipamentos mais sofisticados e precisos para análise dos dados permitiram o desenvolvimento de projetos e pesquisas com maior precisão de análise. A parte 2 do artigo apresenta um pouco da história e dos trabalhos desenvolvidos no laboratório após a aquisição destes equipamentos.

\section{Cinemática e cinética}

A biomecânica pode ser dividida em quatro grandes áreas: estática e dinâmica, cinemática e cinética. A estática é o estudo de sistemas que estão em movimento constante, ou seja, em repouso (sem movimento) ou em movimento a uma velocidade constante. A dinâmica é o estudo de sistemas nos quais a aceleração está presente (HALL, 2016).

A cinemática fornece informações sobre os movimentos realizados, sem referência às forças que causam ou resultam do movimento, de forma que a descrição cinemática dos movimentos pela cinemetria tem sido a técnica mais utilizada para avaliação do movimento. Enquanto a cinemática descreve a aparência do movimento, a cinética é o estudo das forças associadas ao movimento. A cinética consegue avaliar por 
meio da dinamometria quais as forças geradas por meio das ações motoras realizadas, que podem ser externas ou internas (HALL, 2016).

As análises cinemáticas e cinéticas tiveram um grande destaque no LABIOMEC e podemos destacar quatro instrumentos para a análise cinemática que foram utilizados ao longo dos anos. Esses instrumentos demonstram a evolução que essas análises tiveram e como o LABIOMEC acompanhou a mesma.

O Vídeo Position Analyzer foi o primeiro equipamento de cinemática utilizado no LABIOMEC. As filmagens eram feitas com fitas de video home system (VHS) e essas imagens eram projetadas quadro a quadro na televisão, onde o equipamento incorporava a imagem projetada com o sistema de coordenadas cartesianas e a partir daí eram identificados os pontos de interesse em cada quadro (Figura 1A).

Já o sistema Home Made Digitizer foi construído no LABIOMEC em 1992 pelos Prof. Dr. Carlos Bolli Mota, Prof. Orion Mello e Luiz Kirchoff com materiais da própria instituição e utilizado na dissertação de mestrado do Prof. Dr. Ivon Chagas da Rocha Junior (ROCHA JUNIOR, 1993). As imagens eram capturadas em câmeras cinematográficas (LOCAM 51) e reproduzidas por um projetor sobre uma mesa digitalizadora e nesta mesa eram marcados manualmente os pontos de interesse (Figura 1B). Esses dados (coordenadas) provenientes da mesa digitalizadora eram registrados no computador por meio de um software comercial (AutoCAD), construindo o modelo espacial bidimensional do sujeito, que calculava a posição do centro de massa e outras variáveis de interesse.

Figura 1 - Sistema de videografia: televisor colorido Panasonic, câmera de vídeo Panasonic, aparelho videocassete Toshiba DV, vídeo position analyzer VPA-1000 (A) e mesa digitalizadora do Home Made Digitalizer (B)

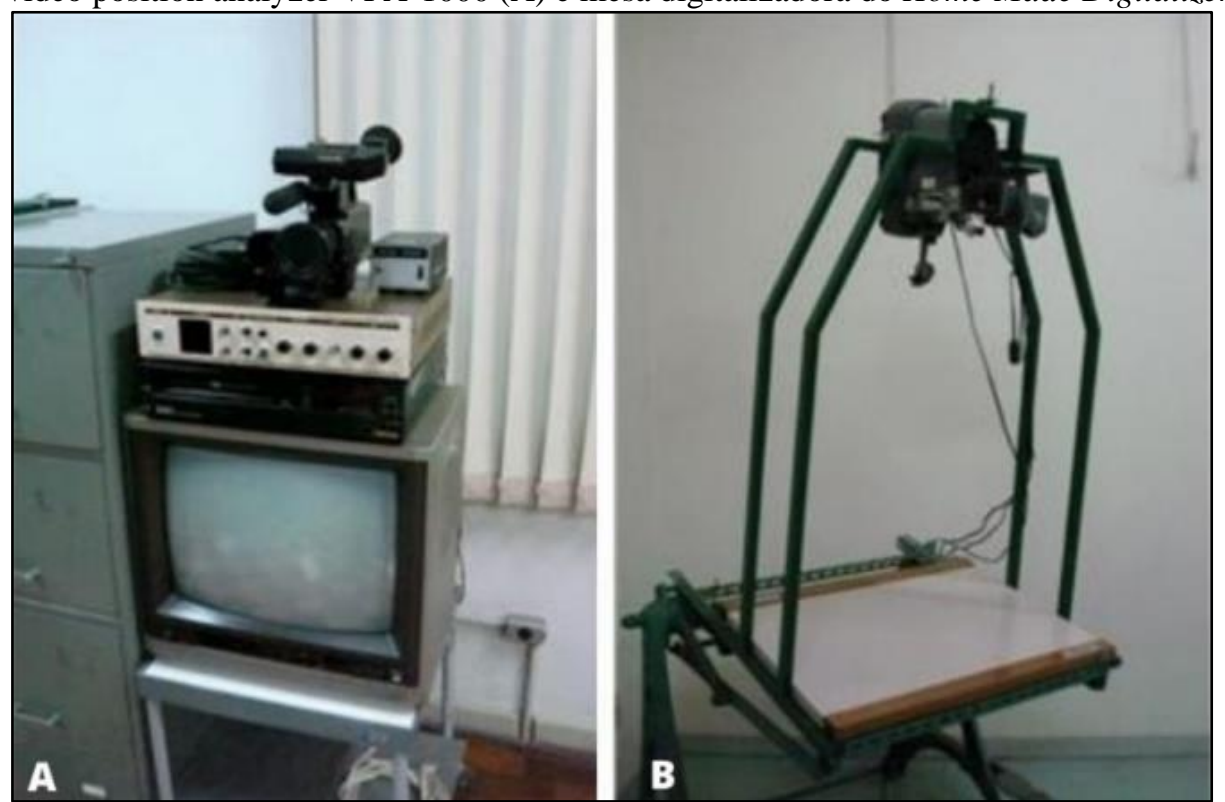

Fonte: Arquivo do LABIOMEC 
O sistema Peak Motus (Peak Performance Inc, USA) foi adquirido pelo LABIOMEC no final da década de 90. Este sistema contava com duas câmeras de vídeo que captavam as imagens para posterior análise. Possibilitava a obtenção de coordenadas tridimensionais a partir de coordenadas bidimensionais das imagens por meio da Reconstrução Linear Direta (Direct Linear Transformation - DLT) (Figura 2), desenvolvida por Abdel-Aziz e Karara em 1971 (ABDEL-AZIZ; KARARA, 2015). Este método possibilitava a obtenção de imagens espaciais (tridimensionais) a partir de coordenadas das imagens, por relações fotogramétricas colineares.

Figura 2 - Computadores para armazenamento (A), sistema de câmeras e calibrador (B), posicionamento das câmeras no laboratório (C) e imagem do calibrador de 17 pontos do sistema de cinemetria Peak Motus (D).

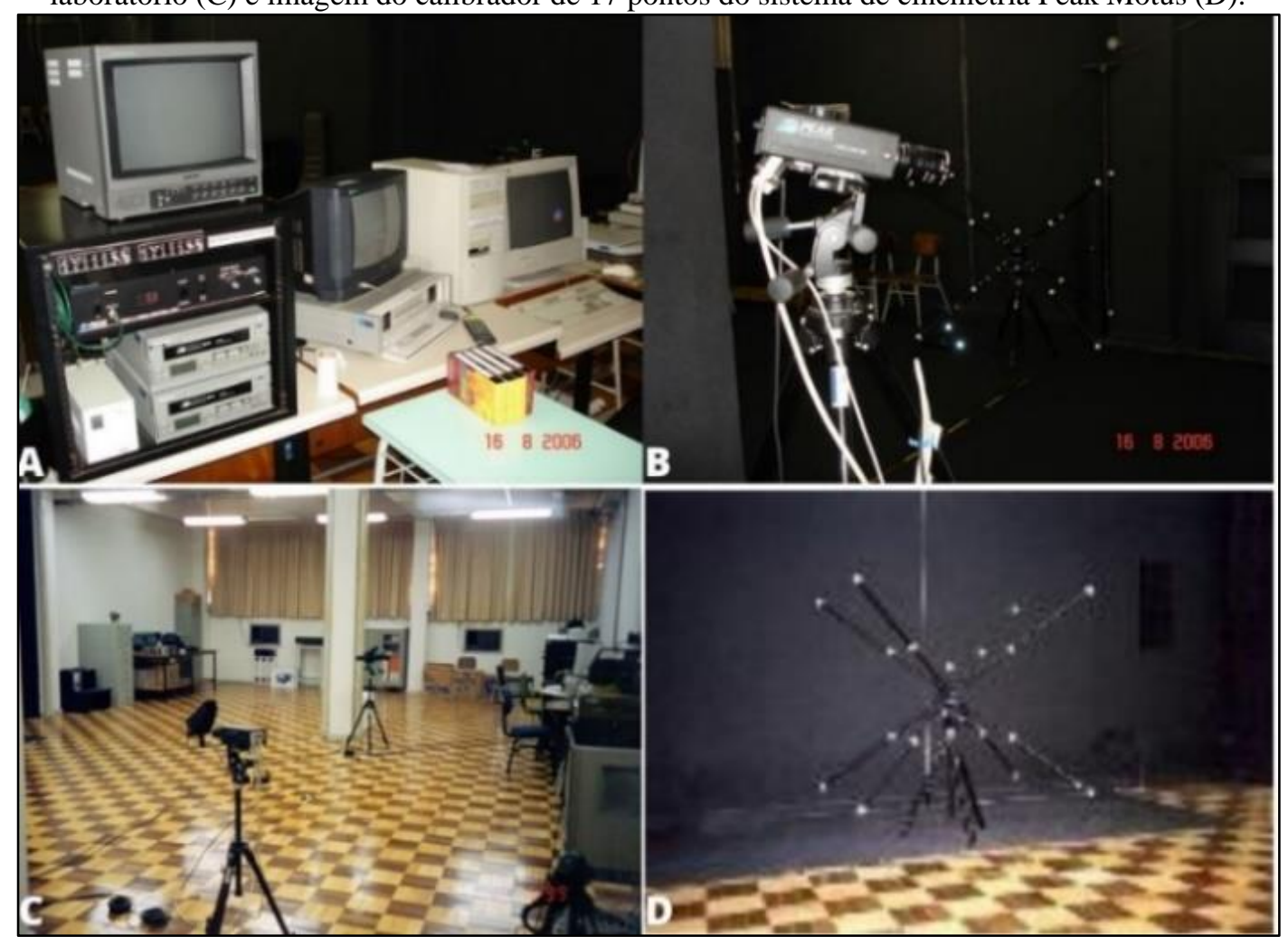

Fonte: Arquivo do LABIOMEC

O LABIOMEC realizou diversos estudos com o sistema Peak Motus, sendo que podemos destacar alguns deles como: estudos com velocistas, seja na saída de bloco (ZANON; ESTRÁZULAS; MOTA, 2002) ou na corrida (PUPO et al., 2006); com ciclistas, seja na cinemática da pedalada de modalidades diferentes (CARPES et al., 2006) ou variações cinemáticas que ocorrem devido a alterações feitas na altura do selim, carga de trabalho ou cadência da pedalada (BINI; TAMBORINDEGUY; MOTA, 2010), estudos com populações especiais, seja com crianças com síndrome de Down (GRAUP et al., 2006) ou com pessoas pós-Acidente Vascular Encefálico (TREVISAN, 2018).. 
Um estudo a ser destacado pela peculiaridade na avaliação é o de Teixeira (2005), o qual investigou a relação entre os movimentos do tronco na marcha humana e no indivíduo montado no cavalo durante o movimento de marcha ao passo. Para esse estudo foi necessário a adaptação do cavalo a ser usado no ambiente do laboratório (Figura 3).

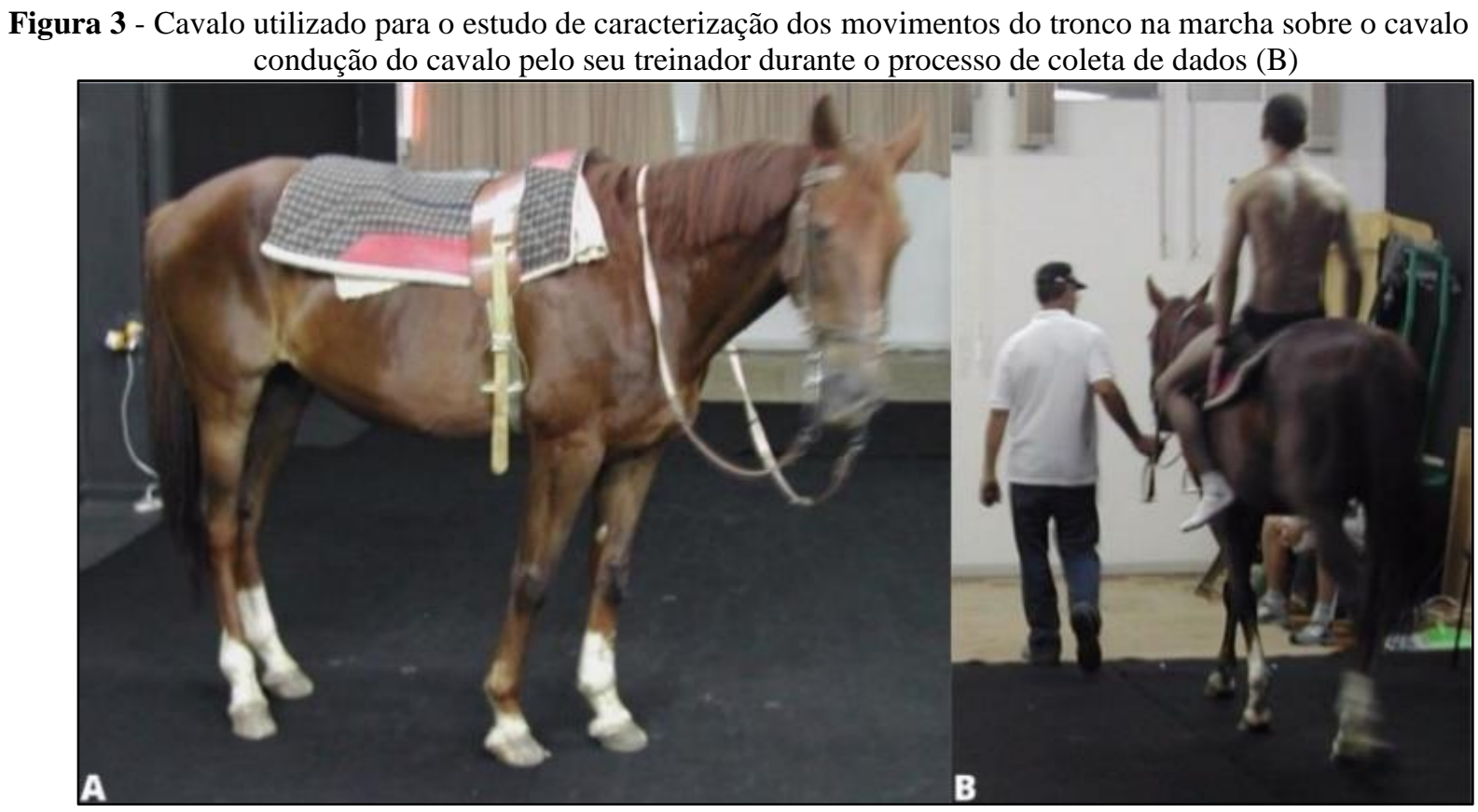

Fonte: TEIXEIRA, 2005

O sistema de cinemetria VICON SYSTEM® foi instalado no LABIOMEC em agosto de 2010, sendo esse o primeiro equipamento do tipo em Universidades Federais no estado do Rio Grande do Sul. Esse sistema é composto por seis câmeras sensíveis ao infravermelho (modelo MX), hardware de controle e um software (Nexus). O sistema pode operar em diferentes frequências de aquisição, reconhecendo em tempo real a posição dos marcadores reflexivos fixados nos sujeitos em pontos anatômicos de referência, rastreando assim os movimentos do corpo e formando os sistemas de referência locais. A partir desses sistemas é possível o cálculo dos movimentos segmentares e articulares, de forma que o posicionamento e o número de marcadores dependem do modelo escolhido para o estudo (Figura 4).

Estudos que já utilizaram esse equipamento no laboratório avaliaram as alterações na marcha, seja ela de pacientes pós reconstrução de ligamento cruzado anterior (STOELBEN; PAPPAS; MOTA, 2019), crianças obesas (PIVETTA; SILVEIRA; MOTA, 2016), idosos (GUADAGNIN et al., 2015), paciente com ataxia (ROSSATO et al., 2016) ou pacientes hemiplégicos (ROSSATO, 2015). 
Figura 4 - Imagem da câmera infravermelha do VICON SYSTEM® (A), marcadores reflexivos fixados em pontos anatômicos do sujeito (B) e coleta de dados da marcha em laboratório (C e D).

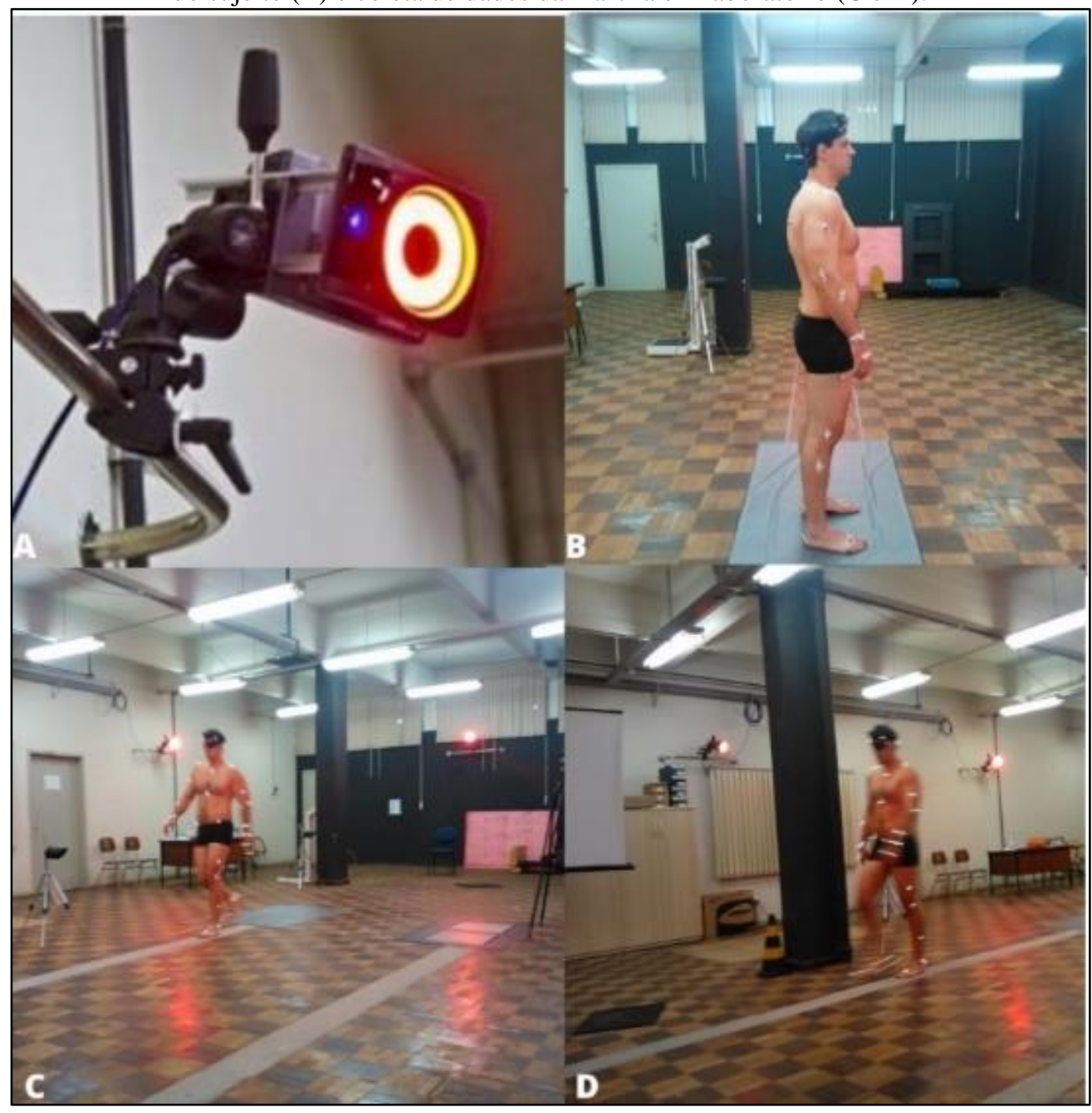

fonte: Arquivo do LABIOMEC

\section{Controle postural}

O controle postural é a habilidade de um indivíduo assumir e manter a posição desejada durante uma atividade estática ou dinâmica (WINTER, 2009). O controle postural pode ser avaliado por meio do comportamento do centro de pressão em equipamentos específicos como a plataforma de força. No LABIOMEC as primeiras plataformas de força chegaram em 2000 e com o aprofundamento dos estudos nessa linha, em 2009 foram adquiridas mais duas plataformas AMTI (Advanced Mechanical Technologies, Inc).

Os primeiros trabalhos de conclusão de curso foram apresentados a partir de 2007 com atletas (PRANKE, 2007), gestantes (MANN, 2007) e em crianças (ALVES, 2008). Entre 2009 e 2012 foram desenvolvidas monografias do curso de Especialização em Atividade Física, Desempenho Motor e Saúde 
organizado pelo CEFD da UFSM e trabalhos em colaboração com outros docentes. Esses estudos avaliaram o efeito de diferentes condições no controle postural como a fadiga em atletas (WIEST, 2009), treinamento de força em idosos (PRANKE, 2009), reabilitação aquática em pacientes com sequelas de acidente vascular encefálico (SOARES, 2010), uso de órtese em paciente com ataxia (ROSSATO, 2012) e a influência do foco atencional no equilíbrio dinâmico de pessoas com dor lombar (POZZOBON, 2012).

Também foram realizados estudos relacionando o equilíbrio postural de adultos jovens em função do sexo (ALVES, 2010), o efeito de treinamento em realidade virtual no controle postural de hemiparéticos (GOMES et al., 2018), efeitos da supressão visual no controle postural (SILVEIRA; PIVETTA; MOTA, 2017a), controle postural entre diferentes esportes (SILVEIRA; PIVETTA; MOTA, 2017b), e a relação entre o índice de arco plantar com o controle postural em mulheres jovens (DORNELES et al., 2014).

A influência do calçado no equilíbrio postural também foi tema investigado em mulheres jovens (MEEREIS, 2011), com uso de bandagens funcionais (COLAZZO, 2017) e em uma dissertação que avaliou a influência do tipo de salto no equilíbrio e centro de gravidade em mulheres jovens (DORNELES; SILVA; MOTA 2015; DORNELES, 2013).

Além da dissertação de mestrado já citada, outras seis foram apresentadas, sendo que a partir de 2015 o LABIOMEC passou a adotar o termo "Controle Postural”. As dissertações do período relacionaramse ao controle postural de idosas com perdas urinárias (RIBEIRO et al., 2019), oscilação corporal em pessoas com e sem dor lombar após caminhada de 10 minutos (MORAIS, 2016), controle postural na execução de teste funcional de membro inferior sem (PETTER, 2017) e com lesão de tornozelo (LOUREIRO, 2018), o efeito da automassagem miofascial (SILVA, 2018) e da prática de ballet clássico no controle postural (PINTO, 2019). A importância da avaliação do controle postural em idosos também é destaque, pois foi tema das teses de doutorado realizadas até o presente momento no Laboratório (LEMOS, 2015; PRANKE, 2015; MEEREIS-LEMOS; GUADAGNIN; MOTA, 2020).

Além de avaliações do controle postural em humanos, o LABIOMEC também colaborou no desenvolvimento de protocolos para avaliação pós-operatória em cães com esse equipamento (Figura 5). 
Figura 5 - Coleta de dados na plataforma de força dos posicionamentos para avaliação de apoio e distribuição do peso nos membros em cães com o animal sendo contido por seu proprietário e avaliado em quatro patas (A), lado direito (B), lado

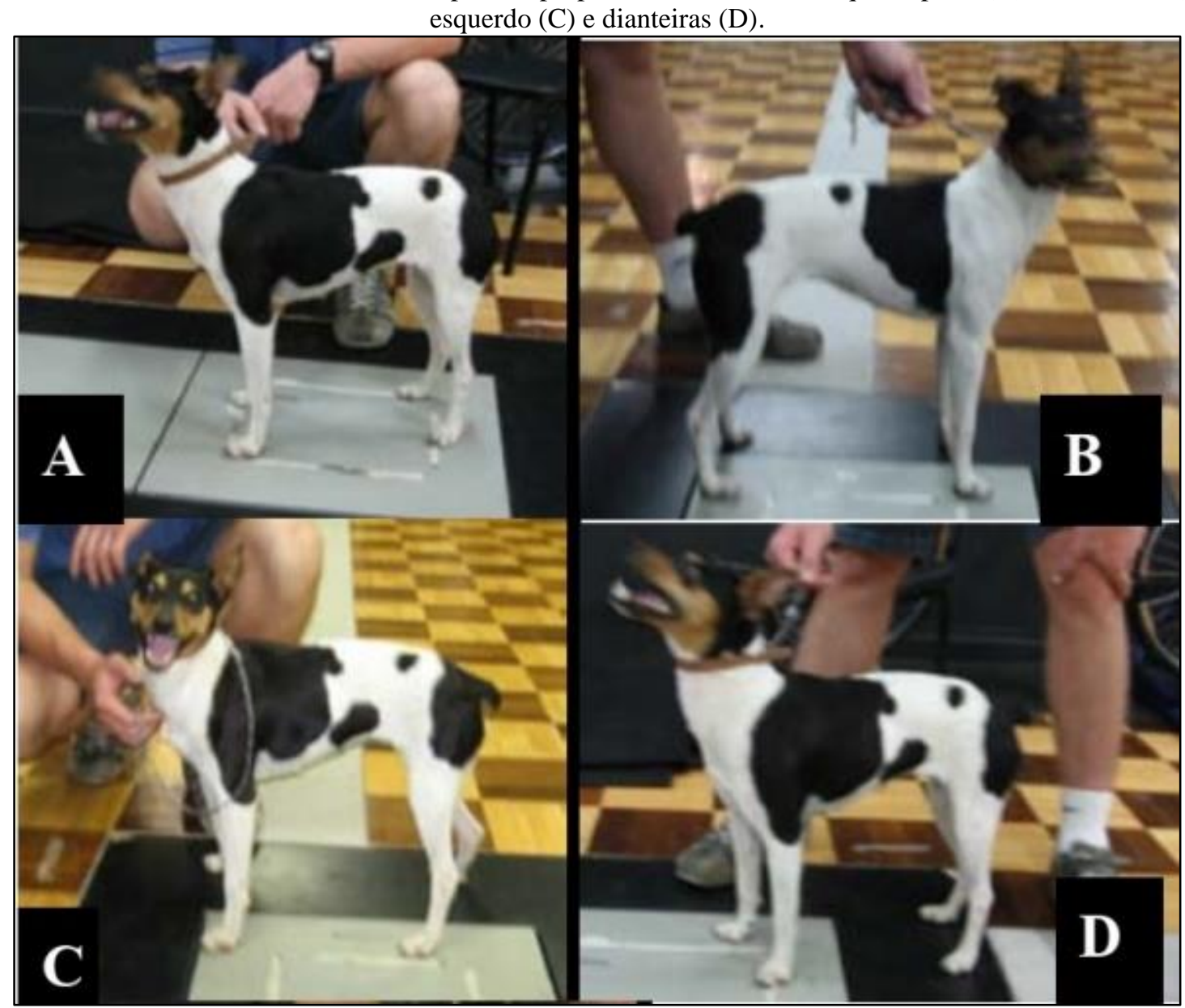

Fonte: BARBOSA; COLS, 2011.

\section{Eletromiografia}

A eletromiografia (EMG) é um conjunto de materiais e métodos responsáveis por registrar a atividade elétrica associada à contração muscular (KONRAD; OWEN; BRIDWELL, 1994). A EMG possibilita a investigação dos músculos utilizados em determinado movimento, do nível de ativação muscular em um gesto, a intensidade e duração do recrutamento muscular, além de possibilitar inferências relativas à fadiga muscular (KONRAD; OWEN; BRIDWELL, 1994; WINTER, 2009).

No LABIOMEC, as análises eletromiográficas iniciaram em 2008 e o primeiro equipamento de EMG foi o eletromiógrafo LYNX System 1200 (Lynx Technologies, São Paulo, Brasil) com 12 canais e frequência de aquisição máxima de $2000 \mathrm{~Hz}$. Os eletrodos utilizados durante o procedimento de coleta de dados eram eletrodos passivos de superfície, com programas de aquisição e processamento específicos: AqDados e AqAnalisys (Figura 6). Os programas e a instrumentação permitiam que a coleta dos dados fosse relativamente rápida, mas o processamento demorado. 
Figura 6 - Programas AqDados (A) e AqDAnalysis (B) para aquisição e processamento dos dados eletromiográficos.

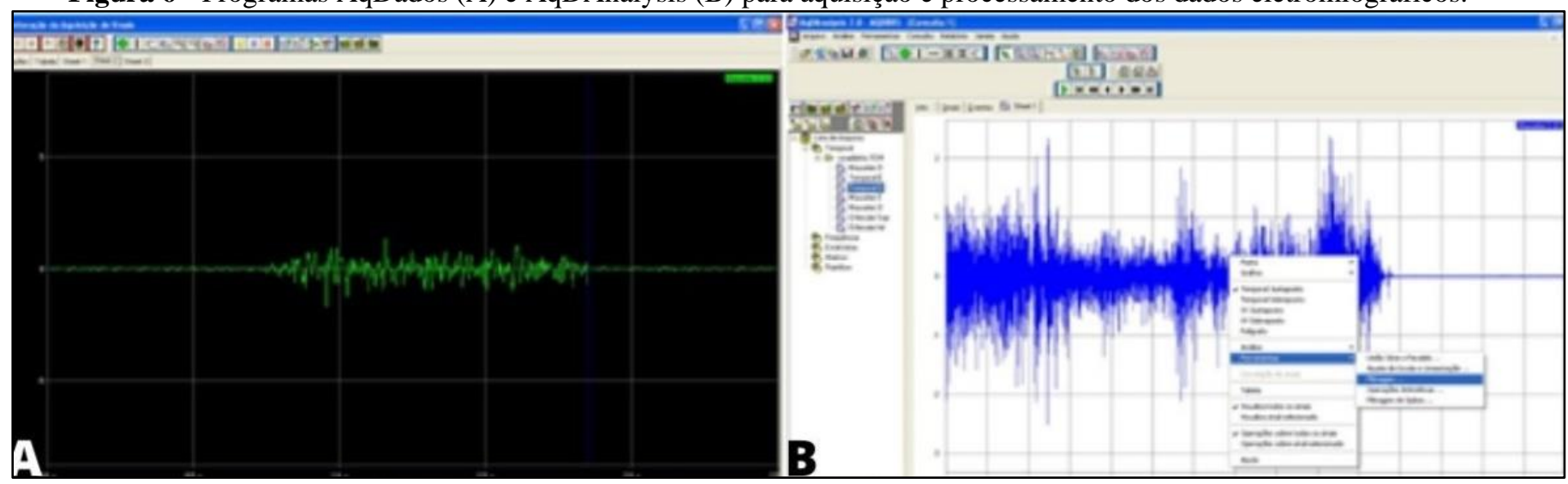

Fonte: Arquivo do LABIOMEC

O equipamento LYNX permitiu a realização de inúmeros estudos da graduação, mestrado e doutorado, tanto de estudantes da UFSM quanto de outras instituições, o que trouxe publicações científicas com grande relevância e a construção de parcerias com outros pesquisadores. Os estudos tiveram diferentes enfoques na área da saúde, com pesquisas sobre: asmáticos (SILVA et al., 2016), mastigação e deglutição; e controle da postura (WIEST et al., 2011). No âmbito esportivo, as análises realizadas no LABIOMEC com a eletromiografia tiveram a temática principal voltada ao ciclismo analisando a ativação dos músculos da coluna lombar (DAGNESE et al., 2015), a assimetria entre membros inferiores (CARPES et al., 2011; DAGNESE et al., 2011), a fadiga e exaustão (CARPES et al., 2010), o ajuste da bicicleta (BINI; TAMBORINDEGUY; MOTA, 2010), dentre outros.

Em 2014, o LABIOMEC adquiriu o sistema TeleMyo Desktop DTS® da NORAXON®, que possui 16 canais e frequência de aquisição atingindo valores de até $3000 \mathrm{~Hz}$. Enquanto o sistema anterior permitia a análise simultânea de oito músculos, esse possibilita a análise de 16 músculos, além de não possuir cabos no par eletrodo/equipamento, o que reduz os ruídos mecânicos e eletromagnéticos da coleta de dados. Apesar do programa MYORESEARCH® ser o usual de coleta de dados, o sistema permite coletas integradas de cinemetria, dinamometria e eletromiografia no VICON SYSTEM® (Figura 7). 
Figura 7 - Coleta de dados com o sistema NORAXON integrado ao VICON SYSTEM® de cinemetria.

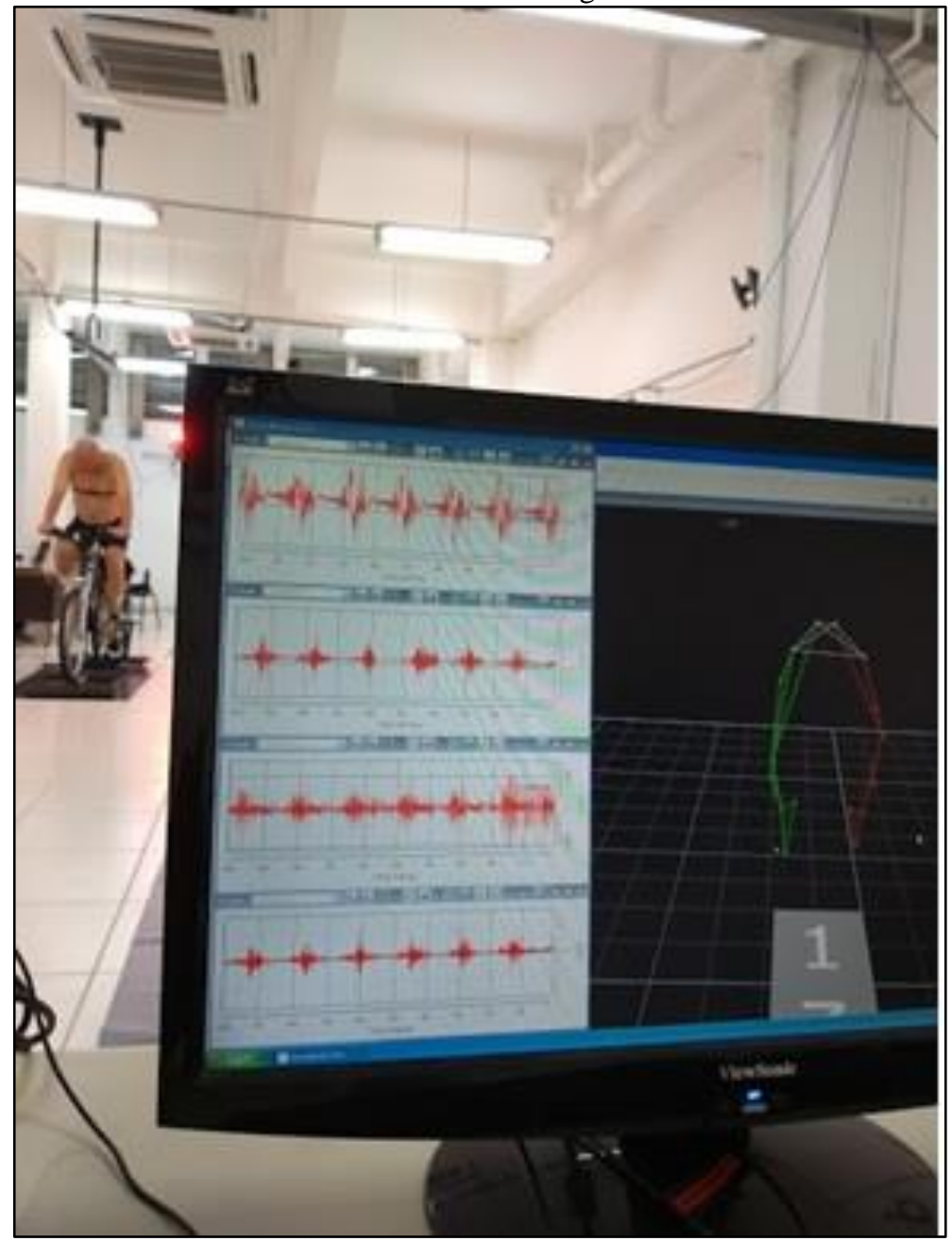

Fonte: Arquivo do Labiomec

Com o equipamento da NORAXON foram realizadas pesquisas na área da saúde e do esporte. Na área da saúde, estudos como a funcionalidade do ombro de mulheres mastectomizadas com e sem reconstrução imediata da prótese (GODOY et al., 2018), estudos do efeito da instrução no valgo dinâmico do joelho em adolescentes com dor patelofemoral (SOUZA, 2019). Na área do esporte, análise do uso de bebidas isotônicas no desempenho de ciclistas (PIVETTA, 2018).

A instrumentação ainda permanece no laboratório, com intuito de avaliar a capacidade de ativação muscular em pesquisas com foco na saúde, no esporte, servindo inclusive como auxílio para aulas práticas ou apresentações formais do próprio laboratório (exemplo: Dia Nacional da Biomecânica). Todas as iniciativas estão envolvidas na busca de respostas, com auxílio da ciência, para melhorar as condições do movimento humano. 


\section{Dia Nacional da Biomecânica}

O Dia Nacional da Biomecânica (National Biomechanics Day-NBD) é um dia de celebração e demonstrações coordenadas em todo o mundo para a valorização da biomecânica entre os jovens de ensino médio. A primeira celebração teve início em 2016 nos Estados Unidos e em 2017 se expandiu para uma celebração internacional (DEVITA, 2018). O objetivo principal é a expansão da biomecânica para sociedade, conscientizando principalmente os jovens da influência e impacto da biomecânica no nosso dia a dia.

O LABIOMEC aderiu ao NBD em 2017, realizando demonstrações práticas das atividades aos alunos do Colégio Técnico Industrial de Santa Maria (CTISM). Neste primeiro ano, após uma palestra sobre as aplicações da biomecânica no dia a dia, os alunos participaram de três oficinas práticas sobre avaliação da pressão plantar, potência no ciclismo e função muscular (FIGURA 8).

Figura 8 - Integrantes do LABIOMEC e oficinas no Colégio Técnico Industrial com os alunos do ensino médio durante o National Biomechanics Day em 2017.

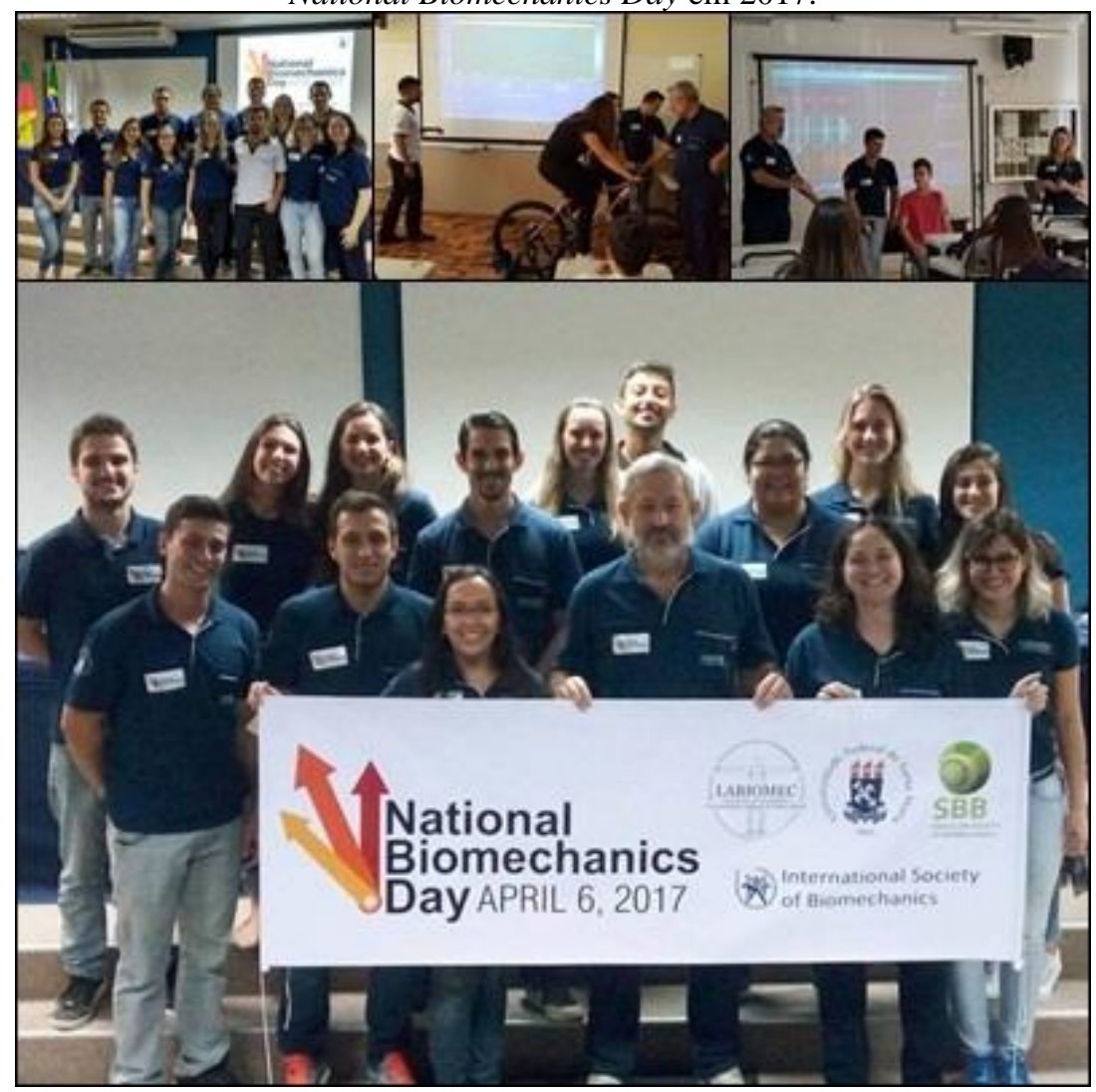

Fonte: Arquivo do LABIOMEC

No ano seguinte, novamente as atividades foram desenvolvidas nas dependências do CTISM. A palestra "Biomecânica aplicada" foi introdutória e apresentou aos alunos a realidade vivenciada nos 
laboratórios e interligada ao cotidiano, bem como as aplicações práticas da biomecânica. Após a aula, os alunos foram divididos em grupos para participarem de quatro oficinas: avaliação da pressão plantar, potência no ciclismo, função muscular e uma oficina de testes funcionais com análise qualitativa da execução de movimentos (Figura 9).

Figura 9 - Integrantes do LABIOMEC e oficinas com os alunos do ensino médio durante o National Biomechanics Day (2018)

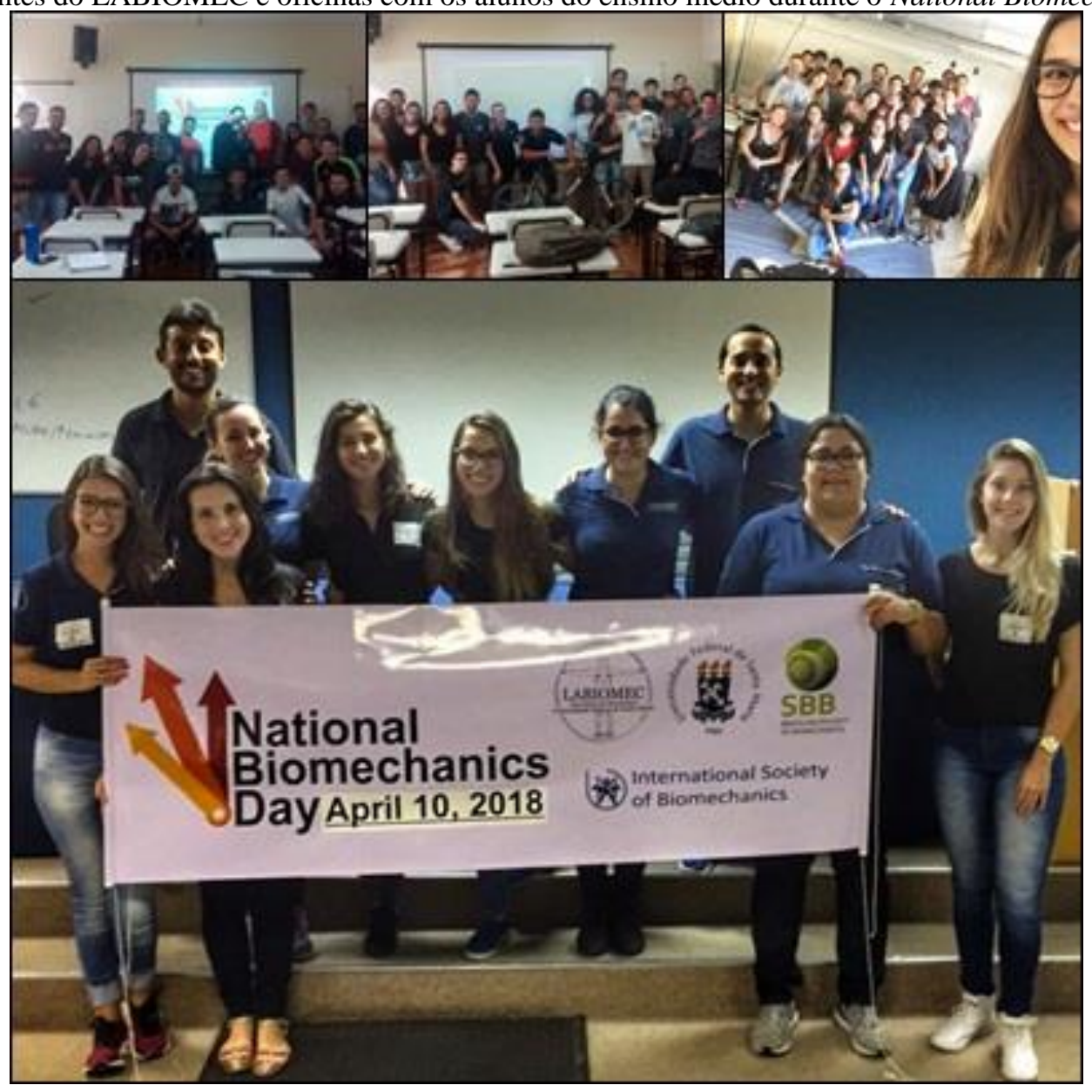

Fonte: Arquivo do LABIOMEC

Em 2019, a atividade foi realizada durante dois dias no LABIOMEC. Novamente, uma palestra inicial sobre o NBD e a apresentação da biomecânica e suas possíveis aplicações. Após essa etapa foram realizadas demonstrações práticas sobre a análise tridimensional do movimento, força e ativação muscular e força de reação do solo (Figura 10). 
Figura 10 - Integrantes do LABIOMEC e alunos ensino médio durante o National Biomechanics Day em 2019.

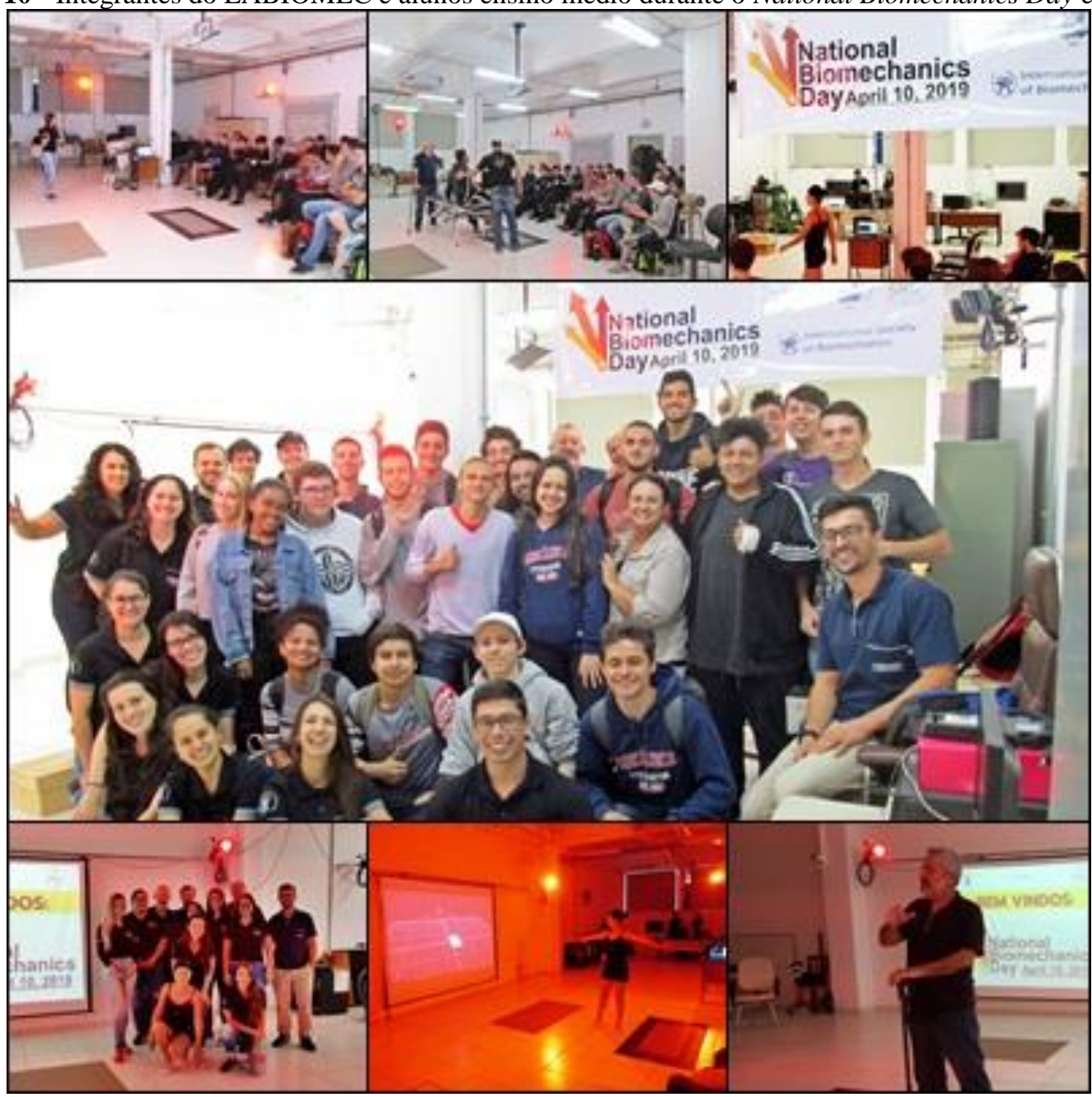

Fonte: Arquivo do LABIOMEC.

Nesses 3 anos de NBD foi possível perceber o interesse e curiosidade dos alunos, principalmente nos momentos de oficinas práticas. Recentemente, uma das alunas do grupo participante em 2017 das atividades iniciou sua graduação em Educação Física na UFSM motivada pelo Dia Nacional de Biomecânica.

\section{Referências}

ABDEL-AZIZ, Y. I, KARARA, D. H. M. Direct linear transformation from comparator coordinates into object space coordinates in close-range photogrammetry. Photogrammetric Engineering and Remote Sensing. v. 81, n. 1, p. 103-107, fev. 2015.

ALVES, R. F. influência do treinamento de futebol no equilíbrio de crianças de seis a nove anos de idade. 2008. 50 f. Trabalho de conclusão do curso (Graduação em Educação Física), Universidade Federal de Santa Maria, Santa Maria, 2008. 
ALVEZ, R. F. Comparação do equilíbrio postural de adultos jovens em função do sexo. $2010.50 \mathrm{f}$. Monografia (Especialização em Atividade física, desempenho motor e saúde), Universidade Federal de Santa Maria, Santa Maria, $2010 . \quad$ Disponível em: http://search.ebscohost.com/login.aspx?direct=true \&db=cat00956a\&AN=ufsm.00073792\&site=eds-live. Acesso em: 30 maio 2020.

BARBOSA, A. L. T et al. Padronização e teste da plataforma de força em padrão ortostático em cães. Arquivo Brasileiro de Medicina Veterinária e Zootecnia. v.63, n.3, 559-66, 2011.

BINI, R. R, ALINE C. T, MOTA, C. B. effects of saddle height, pedaling cadence, and workload on joint kinetics and kinematics during cycling. Journal of sport rehabilitation, v.19, n. 3, p. 301-14, 2010.

CARPES, F. P. et al. Características cinemáticas da pedalada em ciclistas competitivos de diferentes modalidades. Revista Portuguesa de Ciências do Desporto, v. 6, n. 1, p. 07-14, 2006. Disponível em: http://www.scielo.mec.pt/scielo.php?script=sci_arttext\&pid=S164505232006000100002\&lng=pt\&nrm=i so\&tlng=pt. Acesso em: 22 jul. 2020.

CARPES, F. P. et al. Does leg preference affect muscle activation and efficiency? Journal of Electromyography and Kinesiology, v. 20, n. 6, p. 1230-36, 2010. Disponível em: http://www.sciencedirect.com/science/article/pii/S1050641110001227. Acesso em: 30 maio 2020.

CARPES, F. P. et al. Influence of Leg Preference on bilateral muscle activation during Cycling. Journal of Sports Sciences, v. 29, n. 2, p.151-59, 2011. Disponível em: https://doi.org/10.1080/02640414.2010.526625. Acesso em: 30 maio 2020.

COLAZZO, C. C. 2017. Influência de diferentes frequências de uso de salto, tipos de bandagens funcionais e condições visuais no equilíbrio postural de mulheres jovens. 2017. $30 \mathrm{f}$. Trabalho de conclusão de curso (Graduação em Educação Física), Universidade Federal de Santa Maria, Santa Maria. 2017.

DAGNESE, F. et al. Effects of a noncircular chainring system on muscle activation during cycling. Journal of Electromyography and Kinesiology, v. 21, n.1, p. 13-17, 2011. Disponível em: http://www.sciencedirect.com/science/article/pii/S1050641110000295. Acesso em: 30 maio 2020.

DAGNESE, F. et al. Avaliação eletromiográfica dos músculos das costas no ciclismo: um estudo de caso. Saúde (Santa Maria), v. 41, n. 2, p. 107-14, 2015.

DEVITA, P. 2018. Why National Biomechanics Day? Journal of Biomechanics, 2018.

DORNELES, P. P. et al. Relação do índice do arco plantar com o equilíbrio postural. Revista Brasileira de Ciência e Movimento. v. 22, p. 114-120, 2014.

DORNELES, P. P; SILVA, F. S; MOTA, C. B. Comparação do equilíbrio postural entre grupos de mulheres com diferentes faixas etárias. Fisioterapia e Pesquisa, v. 2, p. 392-97, 2015.

DORNELES, P. P. Análise do índice do arco plantar, equilíbrio postural e frequência do uso do salto alto em mulheres de diferentes faixas etárias. 2013. 100 f. Dissertação (Mestrado em Ciências do Movimento Humano), Universidade Federal do Rio Grande do Sul, Porto Alegre, 2013. 
GODOY, A. S. et al. Existe diferença na ordem de ativação dos músculos do ombro entre mulheres com e sem reconstrução mamária após mastectomia? In: IX SIMPÓSIO DE NEUROMECÂNICA APLICADA, IX, 2018, FAMES: Santa Maria-RS.

GOMES, A. G et al. Realidade virtual em hemiparéticos crônicos: efeitos do treino no controle postural. Fisioterapia Brasil. 2018. v. 19, n. 2, 2018.

GRAUP, S. et al. Efeito da equoterapia sobre o padrão motor da marcha cm crianças com síndrome de down: uma análise biomecânica. EF Deportes, Revista Digital, Buenos Aires v.11, n. 96, 2006.

GUADAGNIN, E. C. et al. Effects of regular exercise and dual tasking on spatial and temporal parameters of obstacle negotiation in elderly women. Gait \& posture, v. 42, n. 3, p. 251-56, 2015.

HALL, S. J. Biomecânica Básica. Rio de Janeiro: Guanabara Koogan. 2016.

KONRAD, P E; OWEN, J. H; BRIDWELL, K. H. magnetic stimulation of the spine to produce lower extremity emg responses: significance of coil position and the presence of bone. Spine, v. 19, n. 24, p. 2812-2818,1994.

LEMOS, E. C.W. M; ELIANE, C. G; MOTA, C. B. Influence of strength training and multicomponent training on the functionality of older adults: systematic review and meta-analysis. Revista Brasileira de Cineantropometria \& Desempenho Humano, v. 22, 2020.

LEMOS, L. F. C. Lombalgia e o equilíbrio corporal de atletas da seleção brasileira feminina de canoagem velocidade. 2011.30 f. Monografia (Especialização em Atividade Física, Desempenho Motor e Saúde), Universidade Federal de Santa Maria, Santa Maria, 2011.

LEMOS, L. F. C. Validação de um teste de dupla-tarefa para idosos: controle postural estático e coordenação motora. 100 f. Tese (Doutorado em Ciências do Movimento Humano), Universidade Federal do Rio Grande do Sul, Porto Alegre, 2015.

LOUREIRO, V. L. F. Contribuições relativas dos momentos articulares no controle postural dinâmico de indivíduos com instabilidade crônica de tornozelo. 2018. 50 f. Dissertação (Mestrado em Reabilitação Funcional), Universidade Federal de Santa Maria, Santa Maria, 2018. Disponível em: http://search.ebscohost.com/login.aspx?direct=true \&db=cat00956a\&AN=ufsm.00099421\&site=eds-live. Acesso em: 30 maio 2020.

MANN, L. Gestação: equilíbrio corporal e dor lombar. 2007. 40 f. Trabaho de Conclusão de Curso (Graduação em Educação Física), Universidade Federal de Santa Maria, Santa Maria. 2007.

MEEREIS, E. C. W. Influência do tipo de calçado na postura e no equilíbrio postural de mulheres jovens. 2011. 50 f. Monografia (Especialização em Atividade Física, Desempenho Motor e Saúde), Universidade Federal de Santa Maria, Santa Maria, 2011. Disponível em: http://search.ebscohost.com/login.aspx?direct=true $\& d b=$ cat00956a \&AN=ufsm.00068090\&site=eds-live. Acesso em: 30 maio 2020.

MORAIS, S. C. V. Avaliação da oscilação corporal em indivíduos com e sem dor lombar após caminhada de 10 minutos. 2016. 50 f. Dissertação (Mestrado em Educação Física), Universidade Federal de Santa Maria, Santa Maria, 2016. Disponível em: http://cascavel.cpd.ufsm.br/tede/tde_busca/arquivo.php?codArquivo=8339. Acesso em: 30 maio 2020 . 
PETTER, G. D. N. Avaliação do controle postural durante a execução do Star Excursion Balance Test. 2017. 40 f. Dissertação (Mestrado em Educação Física), Universidade Federal de Santa Maria, Santa Maria, 2017.2 Disponível em: http://search.ebscohost.com/login.aspx?direct=true \&db=cat00956a\&AN=ufsm.00092414\&site=eds-live. Acesso em: 30 maio 2020.

PINTO, K. M. Efeito da prática de ballet clássico no controle postural de escolares. 2019. 50 f. Dissertação (Mestrado em Educação Física), Universidade Federal de Santa Maria, Santa Maria, 2019. Disponível em: http://search.ebscohost.com/login.aspx direct=true $\& d b=$ cat00956a $\& A N=$ ufsm.00104980\&site=eds-live. Acesso em: 30 maio 2020.

PIVETTA, F. M., M. C. SILVEIRA, MOTA, C. B. Comparação dos parâmetros espaço temporais da marcha entre crianças obesas e eutróficas: estudo piloto. Revista Brasileira de Ciência e Movimento, $\mathrm{v}$. 24, n. 1, p. 127-33, 2016.

PIVETTA, F. M. O efeito da bebida isotônica no desempenho de ciclistas recreativos. 2018. $50 \mathrm{f}$. Dissertação (Mestrado em Educação Física), Universidade Federal de Santa Maria, Santa Maria, 2018. Disponível em: http://repositorio.ufsm.br/handle/1/18925. Acesso em: 30 maio 2020.

POZZOBON, D. Influência do foco atencional no equilíbrio dinâmico de pessoas com dor lombar. 2012. 40 f. Monografia (Especialização em Atividade Física, Desempenho Motor e Saúde), Universidade Federal de Santa Maria, Santa Maria, 2012.

PRANKE, G. I. Flexibilidade e equilíbrio corporal em atletas de futsal. 2007. 30 f. Trabalho de conclusão de curso (Graduação em Educação Física), Universidade Federal de Santa Maria, Santa Maria, 2007.

PRANKE, G. I. Influência de um treinamento de força no equilíbrio postural, força muscular e composição corporal de idosos. 2009. 40 f. Monografia (Especialização em Atividade Física, Desempenho Motor e Saúde), Universidade Federal de Santa Maria, Santa Maria, 2009. Disponível em: http://search.ebscohost.com/login.aspx?direct=true \&db=cat00956a\&AN=ufsm.00073942\&site=eds-live. Acesso em: 30 maio 2020.

PRANKE, G. I.. Efeito de Tarefas Cognitivas No Controle Postural Estático e Dinâmico de Adultas Jovens e Idosas Caidoras e Não-Caidoras. 2015. 100 f. Tese (Doutorado em Ciências do Movimento Humano), Universidade Federal do Rio Grande do Sul, Porto Alegre, 2015.

PUPO, J. D. et al. Correlações entre variáveis cinemáticas de velocistas em corridas de velocidade. Lecturas Educación Física y Deportes Revista Digital, v.11, n. 102, Buenos Aires, 2006.

RIBEIRO, J. S. et al. Influência da visão e da dupla tarefa no controle postural de idosas com perdas urinárias. Fisioterapia Brasil, n.20, p. 409-17, 2019.

ROCHA JUNIOR, I. C. D. Análise cinemática do salto em distância: fase final da corrida e impulsão. 1993. 55 f. Dissertação (Mestrado em Ciência do Movimento Humano), Universidade Federal de Santa Maria, Santa Maria, 1993. Disponível em: 
http://search.ebscohost.com/login.aspx?direct=true \&db=cat00956a\&AN=ufsm.699606\&site=eds-live. Acesso em: 30 maio 2020.

ROSSATO, C. E. Avaliação do equilíbrio postural estático e dinâmico de um sujeito com ataxia fazendo uso de órteses. 2012. $40 \mathrm{f}$. Trabalho de conclusão de curso (Graduação em Educação Física), Universidade Federal de Santa Maria, Santa Maria, 2012.

ROSSATO, C. E. et al. Efeito da adição de carga no tronco sobre a marcha de um sujeito com ataxia. Saúde (Santa Maria), Santa Maria, v. 42, n.1, p. 129-136, jan./jun. 2016

ROSSATO, C. E. Análise da mecânica articular durante a marcha de hemiplégicos pós acidente vascular cerebral. 2015. 55 f. Dissertação (Mestrado em Educação Física), Universidade Federal de Santa Maria, Santa Maria, 2015.

SILVA, F. S. D. Influência da automassagem miofascial sobre o controle postural dinâmico e stiffness vertical nos membros inferiores de indivíduos saudáveis. 2018. 55 f. Dissertação (Mestrado em Educação Física), Universidade Federal de Santa Maria, Santa Maria,2018. Disponível em: http://search.ebscohost.com/login.aspx?direct=true \&db=cat00956a\&AN=ufsm.00100321\&site=eds-live. Acesso em: 30 maio 2020.

SILVA, M. C. R. et al. Atividade muscular acessória da respiração após programa de reeducação respiratória e natação em asmáticos. Revista Portuguesa de Ciências do Desporto, v. 16, n. 3, p. 20 -32. 2016.

SILVEIRA, M. C; PIVETTA, F, M; MOTA, C. B. Adultos fisicamente ativos podem controlar seu equilíbrio mesmo em supressão visual. Revista Perspectiva: Ciência e Saúde, v. 2, p. 4-12, 2017 a.

SILVEIRA, M. C; PIVETTA, F, M; MOTA, C. B. Atletas de Diferentes Esportes Não Apresentam Padrões Distintos de Equilíbrio. Revista Perspectiva: Ciência e Saúde, v. 2, p. 43-49. 2017b.

SOARES, J. C. Influência de um programa de reabilitação aquática no equilíbrio postural de indivíduos com seqüelas de acidente vascular encefálico. 2010. 40 f. Monografia (Especialização em Atividade Física, Desempenho Motor e Saúde), Universidade Federal de Santa Maria, Santa Maria, 2010. Disponível em: http://search.ebscohost.com/login.aspx?direct=true $\& d b=$ cat00956a\&AN=ufsm.00073632\&site=eds-live. Acesso em: 30 maio 2020.

SOUZA, G. D. S. D. Influência da instrução na correção do valgo dinâmico do joelho e na ativação muscular do glúteo médio em adolescentes com dor patelofemoral. 2019. $50 \mathrm{f}$. Dissertação (Mestrado em Reabilitação Funcional), Universidade Federal de Santa Maria, Santa Maria, 2019. Disponível em: http://search.ebscohost.com/login.aspx?direct=true \&db=cat00956a\&AN=ufsm.00104069\&site=eds-live. Acesso em: 30 maio 2020.

STOELBEN, K. J. V; EVANGELOS P; CARLOS, B. M. Lower extremity joint moments throughout gait at two speeds more than 4 years after ACL reconstruction. Gait \& posture, v. 70, p. 347-54. 2019.

TEIXEIRA, J. S. Caraterização dos movimentos do tronco na marcha e sobre o cavalo ao passo: um estudo cinemático comparativo. 2005. 46 f. Monografia (Especialização em Ciência do Movimento Humano), Universidade Federal de Santa Maria, Santa Maria, 2005. 
TREVISAN, C. M. et al. Reabilitação da hemiparesia pós-acidente vascular encefálico com mirror visual feedback. Fisioterapia Brasil, v. 8, n. 6, p. 452, 2018.

WIEST, M. J. et al. Changes in postural stability following strenuous running and cycling. Journal of Physical Education and Sport, v. 11, p. 406-413, 2011.

WIEST, M. J. Alterações no controle postural resultantes de exercícios extenuantes de membros inferiores. 2009. Monografia (Educação Física), Universidade Federal de Santa Maria, Santa Maria, 2009.

WINTER, D. A. Biomechanics and Motor Control of Human Movement Biomechanics and Motor Control of Human Movement. 4ed. Estados Unidos: John Wiley \& Sons, 2009. 370 p.

ZANON, S; ESTRÁZULAS, J. A; MOTA, C. B. Análise cinemática da posição de saída dos 100m rasos. Resumo. Este estudo teve como objetivo. Revista Kinesis, Santa Maria, p. 28-186, 2002.

\section{Como citar este artigo}

MOTA, C B; SOARES, J. C; ROSSATO, C. E; PIVETTA, F. M; SILVA, M. C. R; SILVEIRA, M. C; SOUZA, G. S; MEEREIS-LEMOS, E. C. W; BARBOSA, I. M.; LÖBELL, R; MACHADO, R. O; SACCOL, M.F. Um resgate histórico do LABIOMEC na história do CEFD 50 anos: parte 2. Revista Kinesis, Santa Maria, Dossiê CEFD 50 anos, p.01-11, 2020.

*O presente trabalho não contou com apoio financeiro de nenhuma natureza para sua realização. 